\title{
Exosome Derived From Traumatic Brain Injury \\ Patients Accelerates Fracture Healing via SMAD7 Signaling Regulation
}

\section{Yuan Xiong}

Wuhan Union Hospital

Ze Lin

Wuhan Union Hospital

Hang Xue

Wuhan Union Hospital

Yiqiang $\mathrm{Hu}$

Wuhan Union Hospital

Tao Yu

Tongji University

\section{Lang Chen}

Wuhan Union Hospital

\section{Adriana C. Panayi}

Harvard Medical School

Wu Zhou

Wuhan Union Hospital

Yun Sun

Wuhan Union Hospital

\section{Faqi Cao}

Wuhan Union Hospital

\section{Ruiyin Zeng}

Wuhan Union Hospital

Guodong Liu

Third Military Medical University Daping Hospital and Research Institute of Surgery

\section{Bobin Mi}

Wuhan Union Hospital

Guohui Liu ( $\square$ liuguohui@hust.edu.cn )

Wuhan Union Hospital 
Keywords: TBI, Fracture, Exosome, miRNA, SMAD7

Posted Date: November 15th, 2021

DOI: https://doi.org/10.21203/rs.3.rs-1063941/v1

License: (c) (1) This work is licensed under a Creative Commons Attribution 4.0 International License. Read Full License 


\section{Abstract}

\section{Background}

Extremity injuries, especially for bone fracture, predominate in warfare, and around one-third of military personnel sustain at least one kind of traumatic brain injury (TBI). The beneficial role of TBI in osteogenic differentiation and fracture healing was found in clinic, however, the underlying mechanism of this interesting finding is elusive. Exosome (Exos) is nanosized extracellular vesicles, and their regulatory role in bone remodeling have attracted accumulative attention.

\section{Methods}

Ultra-high speed gradient centrifugation was used to extract exosomes (Exos) both from fracture patients combined with TBI (TBI-Exos), and from isolated fracture patients (Ctr-Exos). Human mesenchymal stem cells (hMSCs) proliferation was checked by qRT-PCR, western blotting, ALP staining, alizarin red staining, and EdU (5-ethynyl-2'-deoxyuridine) assays. In addition, C57BL/6J mice were randomly divided into different groups according to the treatments, and the radiographic and histopathological results were analyzed to evaluate the effect of TBI-Exos on fracture healing.

Results

In vitro, suppression of SMAD7 promoted osteogenic differentiation, whereas knock down of miR-21-5p in TBI-Exos deprived this bone-beneficial effect at a great extent. Similarly, our results confirmed that preinjection of TBI-Exos leads to enhanced bone formation, whereas knock down of exosomal miR-21-5p was capable to markedly impair this bone-beneficial effect in vivo.

Conclusion

Our findings provide a potential mechanism of the beneficial role of TBI in fracture healing with a particular focus on the TBI-Exos, and suggest that the use of nanosized materials combined with miR-21$5 p$-mimics may be a promising therapeutic approach to enhance fracture healing in the future.

\section{Background}

Bone fracture is one of the most common injuries encountered in military personnels, which may lead to morbidity and long rehabilitation training period [1]. The incidence of traumatic brain injury (TBI) is also prevalent in the military with a high admission rate of $24.6-41.8$ per 10,000 soldier-years [2]. Interestingly, patients combined with fracture and TBI have long been observed to have a obvious short healing period than the isolated fracture patients. And prior studies have reported a potential relationship between TBI and rapid fracture healing [3-4]. However, the potential mechanisms underlying this interesting phenomenon remain unknown. 
Exosomes (Exos), a promising type of bioactive nanomaterials, are capable to exert their regulatory function through release of the active cargo including exosomal miRNA, which can bind with target mRNAs to modulate their expression rate and degradation with rare interference by the internal and external environmental factors [5]. Currently, the regulatory role of Exos in bone homeostasis have attracted considerable attention [6]. A recent study reports that Exos derived from endothelial cells are capable to reverse osteoporotic activity in vitro and in vivo with a high efficient bone targeting, and provides a potential application of Exos as a bone targeting nanomedicine in reversing bone resorption disorders [7]. Similarly, a prior study indicated that osteoclast was capable to directly affect osteoblastic differentiation via deliver of exosomal miR-214-3p, suggesting that knock-down of miR-214-3p in osteoclasts may be an effective way to ameliorate bone-impaired skeletal disorders [8].

Thus, in this study, we sought to elucidate the beneficial role of TBI in fracture healing with a particular focus on the TBI-Exos. We demonstrate that miR-21-5p is markedly enriched in TBI-Exos, which can be delivered to hMSCs wherein it binds with SMAD7 to enhance osteoblastic differentiation and fracture healing.

\section{Methods}

\section{hMSCs isolated, culture, and transfection}

Firstly, $5 \mathrm{~mL}$ of bone marrow was extracted under sterile condition, and diluted with equal volume of PBS, and then centrifuged at $1500 \mathrm{rpm}$ for 10 minutes at room temperature. Next, the fat layer was discarded, and the diluted marrow solution was slowly added to the equal volume of Ficoll separation solution $(1.077 \mathrm{~g} / \mathrm{mL})$, and centrifuged at $2500 \mathrm{rpm}$ for 30 minutes at room temperature. After which the mononuclear cells were suspended with phosphate buffered solution (PBS) and centrifuged at 1500rpm for 10 minutes at room temperature. Subsequently, the cells were suspended in special medium for hMSCs (\#HUXMA-9001c, Cyagen, Guangzhou, China) and cultured in an incubator at $37^{\circ} \mathrm{C}$ and $5 \% \mathrm{CO}_{2}$. When the cell growth reached $80 \%-90 \%$ fusion, the trypsin-EDTA solution (0.25\%:0.02\%) was used for cell collection. Small interfering RNAs (siRNAs) were designed and constructed by GenePharma company (Soochow, China). Silencing efficiency of the target gene was verified by qRT-PCR and Western blotting analysis.

\section{Exos isolation and examination}

Firstly, the collected samples were moved to a centrifuge tube and centrifuged for 30 minutes at $2000 \times \mathrm{g}$, $4{ }^{\circ} \mathrm{C}$. Then the obtained cell supernatant was moved to a new centrifuge tube and centrifuged for $45 \mathrm{~min}$ at $10,000 \times \mathrm{g}, 4^{\circ} \mathrm{C}$. After the large extracellular vesicles were removed, the supernatant was collected. Next, The filtration step was performed using $0.45 \mu \mathrm{m}$ membrane and the filtered fluid collected and centrifuged for $70 \mathrm{~min}$ at $100,000 \times \mathrm{g}$ at $4{ }^{\circ} \mathrm{C}$. Finally, the supernatant was removed and resuspended in $100 \mu \mathrm{L}$ pre-cooled PBS. $10 \mu \mathrm{L}$ Exos were used for measuring the particle size by Nanoparticle Tracking Analysis (NTA), $10 \mu \mathrm{L}$ Exos were used for exosomal biomarker analysis by Nano- 
Flow-Cytometry (Nano-FCM), and $20 \mu \mathrm{L}$ Exos were used for Exos shape detection by Scanning Electron Microscope (SEM). The remaining Exos were stored at $-80^{\circ} \mathrm{C}$ for the subsequent experiments.

\section{Exos uptake assay}

Firstly, The Exos dye PKH26 (Sigma-Aldrich, MO, united states) was used for detection of the purified Exos in hMSCs. Next, $20 \mathrm{~mL}$ of PBS was used to wash the Exos twice. The hMSCs uptake of the labeled Exos was captured by measuring the immunofluorescence (Red is for Exos, and Blue is for the cell nucleus).

\section{qRT-PCR analysis}

Firstly, TRIZOL was used to extract RNA from the samples, and after which the First strand CDNA was synthesized using M-MLV Reverse Transcriptase kit (\#EQ002, ELK Biotechnology, Wuhan, China). Next, the Real-time quantitative PCR was performed using the EnTurbo ${ }^{\text {TM }}$ SYBR Green PCR SuperMix kit (\#EQ001, ELK Biotechnology, Wuhan, China), and each sample was repeated with three multiple wells. The $2^{-\triangle \Delta C t}$ method was used to calculate the relative mRNA expression. The primers are as follow: miR21-5p, Forward Primer: 5'-GCCCGCTAGCTTATCAGA CTGATG-3', Reverse Primer: 5'-

GTGCAGGGTCCGAGGT-3'; GADPH, Forward Primer: 5'-CCGTTGAATTTGCCGTGA-3'; Reverse Primer: 5'TGATGACCCTTTTGGCTCCC-3'.

\section{Western blotting}

First, the total cell protein extraction reagent was added to extract the protein, and the protein concentration of the sample was measured using the BCA protein concentration determination kit. Then, SDS-PAGE electrophoresis was performed to determine the loading amount according to the sample concentration, ensuring that the total protein loading amount of each sample was $40 \mu \mathrm{g}$. The PVDF membrane was activated with methanol for 3 minutes before use. The membrane transfer time was adjusted according to the molecular weight of the target protein. Next, antibody incubation was carried out, and the transferred membrane was added to the blocking solution at room temperature for sealing for 1 hour. After which the blocking solution was removed, and the primary antibody was added to the solution at $4{ }^{\circ} \mathrm{C}$ and incubated overnight with anti-Collagen I(\#ab34710, Abcam, Cambridge, UK), antiRunx2 (\#ab76956, Abcam, Cambridge, UK), anti-Osteocalcin (\#ab133612, Abcam, Cambridge, UK), antiSMAD7 (\#ab111523, Abcam, Cambridge, UK), and anti- $\beta$-actin (\#ab8227, Abcam, Cambridge, UK). The diluted primary antibody was recovered and washed three times with TBST for 5 minutes each time. The diluted secondary antibody was then added, incubated at room temperature for 30 minutes, and washed with TBST on a shaker at room temperature for 4 times (5 minutes each time). Finally, chemiluminescence detection was carried out.

\section{EdU assay}


hMSCs proliferation rate was assessed using the EDU assay kit (RiboBio, Guangzhou, China) as a prior study [9]. In brief, hMSCs were seeded into a 24-well plate at a cell density of $2.0 \times 10^{4}$ cells/well and cultured for 24 hours before addition of EdU (50 mM). After which the Apollo and

DNA stains were added in to the medium. Finally, the Edu assay images were captured using the fluorescence microscopy (Carl Zeiss, Jena, Germany).

\section{ALP and alizarin red staining}

An alkaline phosphatase color development kit (\#C3206; Beyotime Biotechnology, Shanghai, China) was used to perform the ALP staining of hMSCs. BCIP/NBT substrate was used to treat cells for 7 days. Colorimetric changes were analyzed using a charge-coupled microscope and absorbance was measured at $405 \mathrm{~nm}$. For alizarin red staining, hMSCs were continuously cultured in 24-well plates in an osteogenic medium (\#MUBMX-03011-440, Cyagen, Guangzhou, China) for 21 days to enhance osteogenesis. Red mineralized nodules were analyzed with a charge-coupled device microscope. Absorbance was measured at $570 \mathrm{~nm}$.

\section{Luciferase assay}

SMAD73'-UTR was designed and inserted into the pMIR-report plasmid. For the luciferase reporter assays, hMSCs were cultured and each transfected with $0.5 \mu \mathrm{g}$ firefly luciferase reporter plasmid, $0.5 \mu \mathrm{g}$ vector, and equal amount of AntagomiR-NC or AntagomiR-21-5p using Lipofectamine 3000 (Invitrogen). hMSCs were analyzed using a luciferase assay kits (Promega, Madison, WI, USA).

\section{Ethic consent}

The patient studies were approved by the Committees of Clinical Ethics in the Union Hospital, Tongji Medical College, Huazhong University of Science and Technology (HUST), and informed consent was achieved from all participants. The animals experiments were approved by the Animal Care and Use Ethical Committee of Tongji Medical College, HUST.

\section{Animal administration and histopathological examination}

The fracture murine model was constructed as the prior study depicted [10]. Briefly, C57BL/6J male mice were purchased from the Center of Experimental Animal, Tongji Medical College, HUST. Pentobarbital sodium (80 mg/kg body weight, 1\%) was used for animal anesthesia. After which a posterolateral incision and blunt separation were performed to expose the femur. Next, a diamond disk was used to make a femoral midsection fracture and the fracture site was fixed using a intramedullary needles with $0.6 \mathrm{~mm}$ diameter. Local injection of PBS $(100 \mu \mathrm{L})$ at the fracture site was set as the control group, and mice received $100 \mu \mathrm{L}$ of Ctr-Exos $(100 \mu \mathrm{g} / \mathrm{mL})$, TBI-Exos $(100 \mu \mathrm{g} / \mathrm{mL})$, or TBI-Exos+AntagomiR-21-5p (100 $\mu \mathrm{g} / \mathrm{mL}$ ) were set as the treatment groups. All the local injection was performed at day 0 , day 4 , and day 7 post-injury. On day 14, half of the mice were sacrificed and the bone samples were collected for histological analysis. Briefly, femurs were fixed with 4\% paraformaldehyde overnight and demineralized 
for 2 weeks. $7 \mu \mathrm{m}$ thick paraffin-embedded tissue samples were prepared and subjected to Alcian Blue/hematoxylin-eosin/ orange G (ABHEOG) staining, CD31 and Ki67 staining as previously depicted $[11,12]$.

\section{X-Rays images}

Small animal X-rays examination was carried on at the Central laboratory of Union Hospital, Tongji Medical College, HUST. Briefly, animals were anesthetized with $1 \%$ of pentobarbital sodium $(80 \mathrm{mg} / \mathrm{kg}$ body weight) and imaged using an in vivo FX PRO imaging system (BRUKER, Karlsruhe, Germany) with a one-minute exposure time.

\section{Micro-computer tomography (Micro-CT) examination}

Mice were scanned using the BRUKER SkyScan 1276 scanner with the following parameters: 2,400 views, five frames/view, $37 \mathrm{kV}$, and $121 \mathrm{~mA}$. 3D construction were performed using CT-Vox software (2.1 version, BRUKER, Karlsruhe, Germany). Bone volume (BV), trabecula volume (TV), BV/TV, and bone mineral density (BMD) were assessed with the CTAN software (1.12 version, BRUKER, Karlsruhe, Germany).

\section{Statistical analysis}

All data are depicted as means \pm SD format. GraphPad Prism 8.0 (GraphPad Software, CA, USA) was used for the statistical analysis. Statistical analysis was performed using Student's t test (two groups) or ANOVA with Tukey's post hoc test (over two groups). $\mathrm{P}<0.05$ was the significance threshold.

\section{Results}

\section{TBI-Exos accelerates fracture healing}

Firstly, we checked if the isolated vesicles from serum is Exos using TEM, NTA, and NanoFCM method. The TEM results showed the vesicles have cup or sphere shape, which is consistent with the prior study (Fig. 1A-B) [13]. Furthermore, the NTA result indicated the diameters of these vesicles range from $30 \mathrm{~nm}$ to $150 \mathrm{~nm}$, which is consistent with the prior study (Fig. 1C-D) [13]. In addition, the NanoFCM result verified that these vesicles contained the exosomal surface markers including CD9, CD63 and CD 81 (Fig. $1 \mathrm{E}-\mathrm{F})$. All these results demonstrated the isolated vesicles were Exos. Next, we checked the therapeutic effect of these two kinds of Exos on murine fracture healing. The mice were randomly divided into three groups according to the different treatments (PBS, Ctr-Exos, and TBI-Exos groups). X-rays and micro-CT examination were performed to assess the process of murine fracture healing, and the TBI-Exos group suggested a markedly larger callus formation with narrower fracture gap compared with the PBS group and Ctr-Exos group (Fig. 2A-B). The CT Analyser results indicated that mice in the TBI-Exos group had a higher BV, TV, BV/TV and BMD compared with mice in the PBS group and Ctr-Exos group (Fig. 2C-D). After fixing with $0.5 \%$ paraformalhyde, bone samples were collected for ABHEOG and Von Kossa staining, and TBI-Exos-treated mice exhibited better bone formation performance and less cartilage formation compared with the PBS group and Ctr-Exos group (Fig. 2E-H). Moreover, CD31 and Ki67 
immunohistochemistry indicated a positive effect on angiogenesis and proliferation in the TBI-Exos (Fig. 2I-J, and Fig. S1).

\section{TBI-Exos promote proliferation and osteogenic differentiation in vitro}

Next, we checked if the two kinds of Exos could be taken up by hMSCs. The Exos were labeled with $\mathrm{PKH} 26$ dye (red) and then added into hMSCs medium. The results of this uptake assay suggested that hMSCs were capable to take up Ctr-Exos and TBI-Exos within 12 hours (Fig. 3A). After verifying the uptake ability of hMSCs, we then investigate the effect of these two Exos on the proliferation of hMSCs using EdU assay. The result showed that TBI-Exos could noticeably promote hMSCs proliferation relative to mice in the PBS- and Ctr-Exos-treated group (Fig. 3B). Furthermore, to test the effect of the two Exos on osteogenic differentiation, we performed western blotting analysis to assess the osteogenic-related proteins including Collagen I, Runx 2 and $O C N$ among the different groups. The result indicated a high level of these proteins in the TBI-Exos-treated group relative to other groups (Fig. 3C). Moreover, ALP staining and alizarin red staining were performed to check the level of extracellular mineral deposition, and the results suggested that TBI-Exos was able to induce an enhanced mineral deposition compared with the other treatments (Fig. 3D-G).

\section{Exosomal miR-21-5p is important for the effect of TBI on fracture healing}

In clinic, we found the superior healing performance in fracture patients combined with TBI relative to the isolated fracture patients (Fig. 4A). It is well documented that exosomal miRNA is an essential element of the Exos' cargo, which have been previously demonstrated to have noticeable regulation in the biological process. Noticeably, miR-21-5p is one of the key osteogenic-related miRNAs and has been reported enriched in TBI-Exos [14-15]. Thus, we both checked the miR-21-5p level in patients' serum and Exos. The results showed that miR-21-5p both in serum and Exos is significantly elevated after TBI (Fig. 4B-C). Furthermore, hMSCs were treated with $50 \mu \mathrm{g} / \mathrm{mL}$ Ctr-Exos or $50 \mu \mathrm{g} / \mathrm{mL}$ TBI-Exos for 1 day, 3days and 5 days, respectively. The qRT-PCR result suggested that the miR-21-5p level is significant higher in TBI-Exostreated cells than in Ctr-Exos-treated cells at the three timepoints (Fig. 4D). Next, to check the miR-21-5p's effect on osteoblast proliferation and differentiation, we transfected hMSCs with AntagomiR-NC or AntagomiR-21-5p. The qRT-PCR result suggested a efficient suppression of AntagomiR-21-5p on the miR21-5p expression (Fig. S2). Next, we checked the effect of the different treatments on the proliferation of hMSCs using EdU assay. The result showed that TBI-Exos could noticeably promote hMSCs proliferation, whereas miR-21-5p inhibition could markedly impaired this pro-proliferation effect of TBI-Exos (Fig. 4E). Similarly, the western blotting result indicated the addition of AntagomiR-21-5p could significantly decreased the TBI-Exos-elevated level of osteogenic-related proteins (Fig. 4F). Furthermore, we investigated the potential down-stream mechanism of miR-21-5p. As shown in Fig. 4G, the potential targets of miR-21-5p were identified using the online predicting tools including TargetScan (version: 7.2, http://www.targetscan.org/vert_72/) and miRDB (http://mirdb.org/), and SMAD7 was identified as a predicted target gene. Moreover, to test the interaction of miR-21-5p and SMAD7, a luciferase reporter assay was performed and the result suggested that miR-21-5p was capable to specifically bind to the 
region of SMAD7 mRNA (Fig. 4H). Subsequently, the western blotting indicated that miR-21-5p inhibition obviously led to a elevation of SMAD7 level (Fig. 4I). To check the role of SMAD7 in regulation of hMSCs proliferation and differentiation, siRNA SMAD7 was constructed. The qRT-PCR result verified the high efficiency of siRNA SMAD7 in suppression of SMAD7 expression, and the addition of AntagomiR-21-5p could partially reverse the reduction of SMAD7 (Fig. $4 \mathrm{~J}$ ). In addition, we checked the effect of SMAD7 on the proliferation of hMSCs using EdU assay, which showed that siRNA SMAD7 could noticeably promote hMSCs proliferation, whereas miR-21-5p inhibition could partially impaired this pro-proliferation effect of siRNA SMAD7 (Fig. 4K). Similarly, the western blotting result indicated the addition of AntagomiR-21-5p could significantly decreased the siRNA SMAD7-elevated level of osteogenic-related proteins (Fig. 4L).

\section{Exosomal miR-21-5p accelerates fracture healing in vivo}

Finally, we checked the therapeutic effect of exosomal miR-21-5p on murine fracture healing. The mice were randomly divided into three groups according to the different treatments (PBS, TBI-Exos, and TBIExos+AntagomiR-21-5p groups). X-rays and micro-CT examination were performed to assess the process of murine fracture healing, and the TBI-Exos group suggested a markedly larger callus formation with narrower fracture gap compared with the PBS group, whereas after the addition of AntagomiR-21-5p, the pro-healing effect of TBI-Exos is partially reversed (Fig. 5A-B). The CT Analyser results indicated that mice in the TBI-Exos group had a higher BV, TV, BV/TV and BMD compared with mice in the PBS group and the addition of AntagomiR-21-5p could partially reverse the TBI-Exos' effect (Fig. 5C-D). After fixing with 0.5\% paraformalhyde, bone samples were collected for ABHEOG and Von Kossa staining. Similarly, TBI-Exostreated mice exhibited better bone formation performance and less cartilage formation compared with the PBS group and the addition of AntagomiR-21-5p was able to partially impair the pro-healing effect of TBI-Exos (Fig. 5E-H). Moreover, CD31 and Ki67 immunohistochemistry indicated a positive effect on angiogenesis and proliferation in the TBI-Exos-treated group, and AntagomiR-21-5p could partially reverse this effect (Fig. 5I-J, and Fig. S3).

\section{Discussion}

Around $5 \%$ of military personnel have suffered stress fracture injuries during the physical training period [16]. There is clear necessity and value to elucidate the interaction between TBI and fracture, which may provide novel insights for accelerated fracture-healing strategy. Prior researches have paid a great attention on the role of bioactive regulators in bone metabolism, with few research identifying the relationship between TBI-related exosomal miRNAs and fracture healing [17-19]. In this study, we identified miR-21-5p is markedly enriched in TBI-Exos compared with Ctr-Exos, and the exosomal miR-21$5 p$ was further demonstrated to accelerate bone remodeling in vitro and in vivo. Furthermore, the suppression of SMAD7 signaling was assumed as the underlying mechanism of this pro-healing effect. Our findings revealed the use of nanomaterials combined with miR-21-5p-mimics may act as a feasible strategy to enhance fracture healing. 
Exos are endogenous nanosized vesicles secreted by a wide array of cells into the extracellular microenvironment [20]. Exos represent an important mode of intercellular communication and serve as a kind of ideal carrier for delivery of active proteins, lipids, and RNA $[21,22]$. Currently, due to their robust regulation of cellular communication in prokaryotes and eukaryotes, Exos attracted increasing research interest [23]. Exosomal miRNA is one of the most important regulators in Exos, and many exosomal miRNAs have previously been reported to modulate bone remodeling $[24,25]$. In our prior study, we demonstrated that exosomal miR-5106 derived from M2 subtype macrophage induces mesenchymal stem cells towards osteoblastic differentiation and promotes fracture healing [26]. Thus, in the present study, we throw a particular sight on the exosomal miRNA derived from TBI-Exos and sought to elucidate the mechanism underlying the pro-healing effect of TBI on fracture healing. Interestingly, our results indicated that TBI-Exos promotes osteogenic differentiation of fractures sites and also stimulate local angiogenesis. Prior studies have demonstrated that alteration of microenvironment induced by the enhanced function of bone mesenchymal stem cells could stimulate angiogenesis through the intercellular communication $[27,28]$. We assume that two issues may contribute to the enhanced angiogenesis in this study. On the one hand, the TBI-Exos may also be taken up by endothelial cells, and the function of endothelial cells could be enhanced by the release of active cargos from TBI-Exos, and thereby promoting angiogenesis in the fracture sites. On the other hand, the TBI-Exos directly mediated the cellular functions of hMSCs and angiogenic endothelial cells and regulated communication between them by promoting paracrine effects via exosomal interactions in vitro. In our next study, we will further investigate the relationship between TBI-Exos and the function of vascular endothelial cell, and verify the effect of TBI-Exos on vascular endothelial cell tube-formation function and angiogenesis at fracture sites.

miR-21-5p is a well-known osteogenic-related miRNA in regulation of bone remodeling [29]. A recent study reported that exosomal miR-21-5p derived from growth hormone-secreting pituitary adenoma induces bone formation in vitro and increases trabecula number in vivo [30]. Furthermore, Dai et al found that miR-21-5p expression was significantly elevated from the acute to the chronic phase of TBI, and neuronal Exos enriched with miR-21-5p was able to attenuate trauma-induced nerve injury [31]. Thus, in this study, we paid attention to check whether the miR-21-5p level was elevated in fracture patients combined with TBI. We found miR-21-5p is markedly enriched in serum and Exos from the fracture combined with TBI patients relative to the isolated fracture patients. Similarly, the elevated miR-21-5p expression was also found in hMSCs treated by TBI-Exos. We then checked the exosomal miR-21-5p on fracture healing in a murine fracture model. The in vivo result demonstrated a noticeable promotion of TBI-Exos on mice femoral fracture healing, and this effect will partially reversed bymiR-21-5p inhibition. Hence, we concluded that exosomal miR-21-5p is the main underlying mechanism of TBl's effect on fracture healing.

Osteoblastic differentiation is essential for bone formation as an important step of the fracture healing [32]. Impairment of osteoblastic differentiation is assumed to be a crucial issue for the disrupted bone remodeling [33]. We identify SMAD7 as a potential downstream target of miR-21-5p. Prior study have welldocumented that SMAD7 served as an inhibitory signal for the TGF- $\beta$ /BMP pathway, and suppressed bone formation in a negative feedback manner [34]. In this study, we found a distinct role of SMAD7 in 
the osteoblastic differentiation. When downregulated, SMAD7 is no longer capable to restrain the osteoblastic differentiation process, and thus resulting in a improved performance of bone remodeling. Furthermore, when adding the miR-21-5p inhibition treatment, the pro-differentiation effect of siRNA SMAD7 was partially reversed. All these results suggested the miR-21-5p/SMAD7 axis in the regulation of osteoblastic differentiation.

Our findings have potential application value for the treatment of diseases related to bone formation disorders. The TBI-Exos-mediated delivery of miR-21-5p with promotion effects on bone mesenchymal stem cells is probably the main contributor to induce osteogenic differentiation. Considering the systemic dysfunction of bone remodeling in patients with bone formation disorders, therapeutic interventions tackling TBI-Exos and miR-21-5p would probably improve bone regeneration for these patients. In this study, we particularly focused on exosomal miR-21-5p delivery from TBI-Exos to hMSCs. However, Exos actually deliver other active factors than miRNAs potentially regulating the target cells. In addition, other regulatory issues, such as angiogenesis and inflammation, may also affect bone remodeling and fracture healing. It would be of necessity to further elucidate the definite factor of TBI-Exos in mediating fracture healing, and uncover the changes of microenvironment of fracture site and their roles in fracture healing.

\section{Declarations}

\section{Ethics approval and consent to participate}

The study was approved by the Ethics Committee of Wuhan Union Hospital.

\section{Consent for publication}

Not applicable

\section{Availability of data and materials}

All data of this study are available within the paper.

\section{Competing interests}

All authors declare no conflict of interest.

\section{Funding}

This work was supported by the National Science Foundation of China (No. 82002313, No. 82072444, 31900963), Hubei Province Key Laboratory of Oral and Maxillofacial Development and Regeneration (No.2020kqhm008), the Health Commission of Hubei Province (No. WJ2019Z009), and the China Postdoctoral Science Foundation (No. 2021TQ0118).

\section{Authors' contributions}


Yuan Xiong: Conceptualization, Writing-original draft. Ze Lin: Investigation, Data curation, Writing-original draft. Hang Xue: Data curation, Writing-original draft. Yiqiang Hu: Writing-original draft and revised the manuscript. Tao Yu: Investigation and formal analysis. Lang Chen: Visualization. Adriana C. Panayi: made critical contributions to the concept and writing of the manuscript. Wu Zhou: Validation. Yun Sun: Technical assistance. Faqi Cao: Formal analysis. Guodong Liu: Investigation. Ruiyin Zeng: Data collection. Guodong Liu: Validation. Bobin Mi: Conception, revised the manuscript. Guohui Liu: Conception, supervision, and writing-original draft.

\section{Acknowledgements}

Yuan Xiong, Ze Lin and Hang Xue contributed equally to this work.

\section{References}

1. Ben-Ami IS, Ankory R, Kadar A, Rotman D, Snir N, Schermann H. The Effect of Previous Methylphenidate Use on Incidence of Stress Fractures in Military Recruits: A Retrospective Cohort. J Bone Joint Surg Am. 2018;100(11):930-935. doi:10.2106/JBJS.17.01267

2. Bhattrai A, Irimia A, Van Horn JD. Neuroimaging of traumatic brain injury in military personnel: An overview. J Clin Neurosci. 2019;70doi:10.1016/j.jocn.2019.07.001

3. Xiong $\mathrm{Y}$, Cao F, Hu L, et al. miRNA-26a-5p Accelerates Healing via Downregulation of PTEN in Fracture Patients with Traumatic Brain Injury. Mol Ther Nucleic Acids. 2019;17:223-234. doi:10.1016/j.omtn.2019.06.001

4. $\mathrm{Hu} \mathrm{L}$, Liu J, Xue H, et al. miRNA-92a-3p regulates osteoblast differentiation in patients with concomitant limb fractures and TBI via IBSP/PI3K-AKT inhibition. Mol Ther Nucleic Acids. 2021;23:13451359. doi:10.1016/j.omtn.2021.02.008

5. Xiong Y, Chen L, Yan C, et al. Circulating Exosomal miR-20b-5p Inhibition Restores Wnt9b Signaling and Reverses Diabetes-Associated Impaired Wound Healing. Small. 2020;16(3):e1904044. doi:10.1002/smll.201904044

6. Zhang D, Wu Y, Li Z, et al. MiR-144-5p, an exosomal miRNA from bone marrow-derived macrophage in type 2 diabetes, impairs bone fracture healing via targeting Smad1. J Nanobiotechnology. 2021;19(1):226. doi:10.1186/s12951-021-00964-8

7. Song H, Li X, Zhao Z, et al. Reversal of Osteoporotic Activity by Endothelial Cell-Secreted Bone Targeting and Biocompatible Exosomes. Nano Lett. 2019;19(5):3040-3048. doi:10.1021/acs.nanolett.9b00287

8. Li D, Liu J, Guo B, et al. Osteoclast-derived exosomal miR-214-3p inhibits osteoblastic bone formation. Nat Commun. 2016;7:10872. doi:10.1038/ncomms10872 
9. Chen L, Xiong Y, Yan C, et al. LncRNA KCNQ10T1 accelerates fracture healing via modulating miR-701-3p/FGFR3 axis. FASEB J. 2020;34(4):5208-5222. doi:10.1096/fj.201901864RR

10. Xiong Y, Yan C, Chen L, et al. IL-10 induces MC3T3-E1 cells differentiation towards osteoblastic fate in murine model. J Cell Mol Med. 2020;24(1):1076-1086. doi:10.1111/jcmm.14832

11. Hou T, Liu Y, Wang X, et al. Ginsenoside Rg1 promotes lymphatic drainage and improves chronic inflammatory arthritis. J Musculoskelet Neuronal Interact. 2020;20(4):526-534.

12. Zohdy M, Abd El Hafez A, Abd Allah MYY, Bessar H, Refat S. Ki67 and CD31 Differential Expression in Cutaneous T-Cell Lymphoma and Its Mimickers: Association with Clinicopathological Criteria and Disease Advancement. Clin Cosmet Investig Dermatol. 2020;13:431-442. doi:10.2147/CCID.S256269

13. He C, Zheng S, Luo Y, Wang B. Exosome Theranostics: Biology and Translational Medicine. Theranostics. 2018;8(1):237-255. doi:10.7150/thno.21945

14. Bemben DA, Baker BS, Buchanan SR, Ade CJ. Circulating MiR-21 expression is upregulated after 30 days of head-down tilt bed rest. Osteoporos Int. 2021;32(7):1369-1378. doi:10.1007/s00198-02005805-2

15. Yin Z, Han Z, Hu T, et al. Neuron-derived exosomes with high miR-21-5p expression promoted polarization of M1 microglia in culture. Brain Behav Immun. 2020;83:270-282. doi:10.1016/j.bbi.2019.11.004

16. Armstrong DW, Rue J-PH, Wilckens JH, Frassica FJ. Stress fracture injury in young military men and women. Bone. 2004;35(3):806-816.

17. Lieben L, Callewaert F, Bouillon R. Bone and metabolism: a complex crosstalk. Horm Res. 2009;71 Suppl 1:134-138. doi:10.1159/000178056

18. Hawkes CP, Mostoufi-Moab S. Fat-bone interaction within the bone marrow milieu: Impact on hematopoiesis and systemic energy metabolism. Bone. 2019;119:57-64. doi:10.1016/j.bone.2018.03.012

19. Lucas S, Omata Y, Hofmann J, et al. Short-chain fatty acids regulate systemic bone mass and protect from pathological bone loss. Nat Commun. 2018;9(1):55. doi:10.1038/s41467-017-02490-4

20. Wortzel I, Dror S, Kenific CM, Lyden D. Exosome-Mediated Metastasis: Communication from a Distance. Dev Cell. 2019;49(3):347-360. doi:10.1016/j.devcel.2019.04.011

21. Milane L, Singh A, Mattheolabakis G, Suresh M, Amiji MM. Exosome mediated communication within the tumor microenvironment. J Control Release. 2015;219:278-294.

doi:10.1016/j.jconrel.2015.06.029 
22. Yue B, Yang H, Wang J, et al. Exosome biogenesis, secretion and function of exosomal miRNAs in skeletal muscle myogenesis. Cell Prolif. 2020;53(7):e12857. doi:10.1111/cpr.12857

23. Li S-P, Lin Z-X, Jiang X-Y, Yu X-Y. Exosomal cargo-loading and synthetic exosome-mimics as potential therapeutic tools. Acta Pharmacol Sin. 2018;39(4):542-551. doi:10.1038/aps.2017.178

24. Su T, Xiao Y, Xiao Y, et al. Bone Marrow Mesenchymal Stem Cells-Derived Exosomal MiR-29b-3p Regulates Aging-Associated Insulin Resistance. ACS Nano. 2019;13(2):2450-2462. doi:10.1021/acsnano.8b09375

25. Li R, Zhao K, Ruan Q, Meng C, Yin F. Bone marrow mesenchymal stem cell-derived exosomal microRNA-124-3p attenuates neurological damage in spinal cord ischemia-reperfusion injury by downregulating Ern1 and promoting M2 macrophage polarization. Arthritis Res Ther. 2020;22(1):75. doi:10.1186/s13075-020-2146-x

26. Xiong Y, Chen L, Yan C, et al. M2 Macrophagy-derived exosomal miRNA-5106 induces bone mesenchymal stem cells towards osteoblastic fate by targeting salt-inducible kinase 2 and $3 . J$ Nanobiotechnology. 2020;18(1):66. doi:10.1186/s12951-020-00622-5

27. Guo Q, Yang J, Chen Y, et al. Salidroside improves angiogenesis-osteogenesis coupling by regulating the HIF-1a/VEGF signalling pathway in the bone environment. Eur J Pharmacol. 2020;884:173394. doi:10.1016/j.ejphar.2020.173394

28. Chen W, Xu K, Tao B, et al. Multilayered coating of titanium implants promotes coupled osteogenesis and angiogenesis in vitro and in vivo. Acta Biomater. 2018;74:489-504. doi:10.1016/j.actbio.2018.04.043

29. Smieszek A, Marcinkowska K, Pielok A, Sikora M, Valihrach L, Marycz K. The Role of miR-21 in Osteoblasts-Osteoclasts Coupling In Vitro. Cells. 2020;9(2)doi:10.3390/cells9020479

30. Xiong $Y$, Tang $Y$, Fan F, et al. Exosomal hsa-miR-21-5p derived from growth hormone-secreting pituitary adenoma promotes abnormal bone formation in acromegaly. Trans/ Res. 2020;215doi:10.1016/j.trsl.2019.07.013

31. Li D, Huang S, Zhu J, et al. Exosomes from MiR-21-5p-Increased Neurons Play a Role in Neuroprotection by Suppressing Rab11a-Mediated Neuronal Autophagy In Vitro After Traumatic Brain Injury. Med Sci Monit. 2019;25:1871-1885. doi:10.12659/MSM.915727

32. Mi B, Xiong Y, Yan C, et al. Methyltransferase-like 3-mediated N6-methyladenosine modification of miR-7212-5p drives osteoblast differentiation and fracture healing. J Cell Mol Med. 2020;24(11):63856396. doi: $10.1111 / \mathrm{jcmm} .15284$

33. Meyer MB, Benkusky NA, Sen B, Rubin J, Pike JW. Epigenetic Plasticity Drives Adipogenic and Osteogenic Differentiation of Marrow-derived Mesenchymal Stem Cells. J Biol Chem. 
34. Zhao F, Ma X, Qiu W, et al. Mesenchymal MACF1 Facilitates SMAD7 Nuclear Translocation to Drive Bone Formation. Cells. 2020;9(3)doi:10.3390/cells9030616

\section{Figures}

A

TBI-Exos

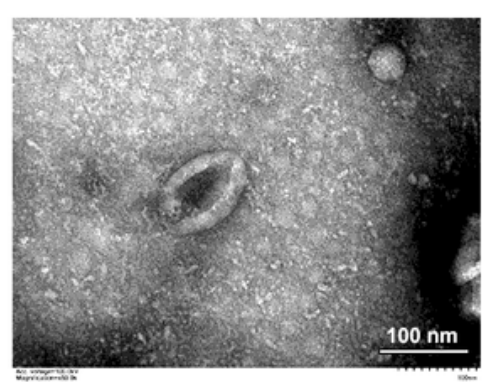

B

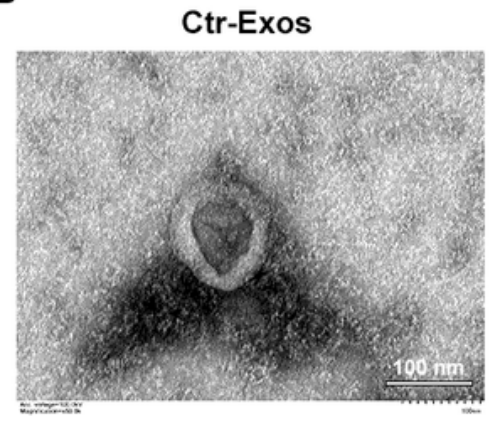

E
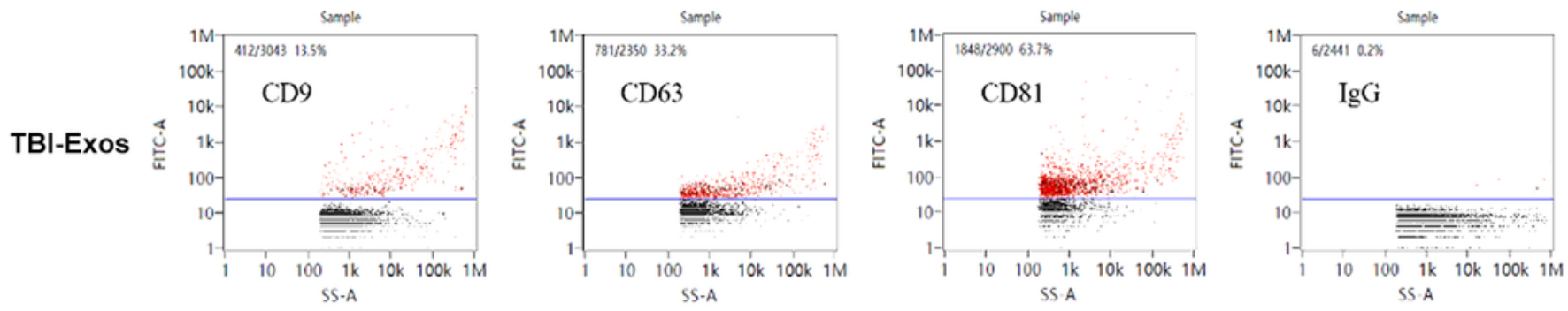

$\mathbf{F}$

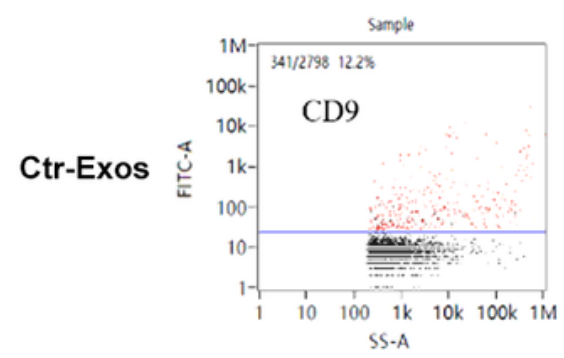

\section{Figure 1}

C
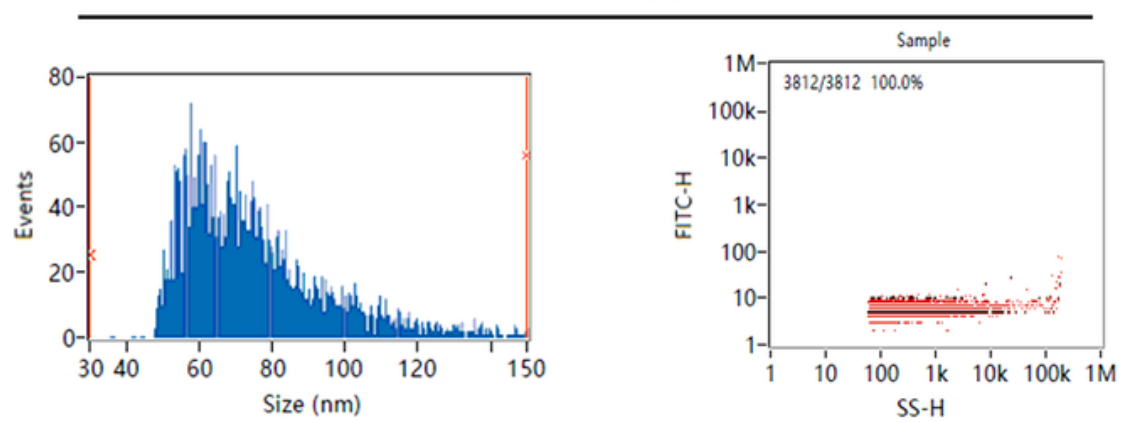

D

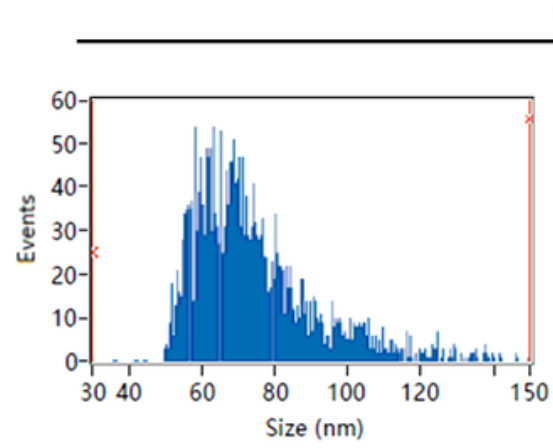

Ctr-Exos

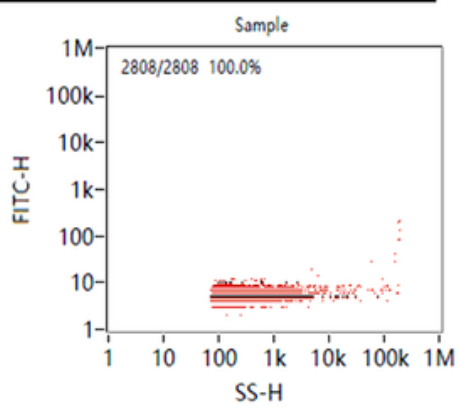


Figure 1

The characteristics of Ctr-Exos and TBI-Exos. A-B. TEM images of Ctr-Exos and TBI-Exos, scale bar: 100 $\mathrm{nm}$. C-D. The size of these nanoparticles was measured by NTA. E-F. The surface markers of Exos including CD9, CD63, and CD81 were detected using NanoFCM.

Fgure 2

A

A

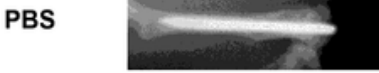

Ctr-Exos

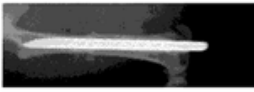

TBI-Exos

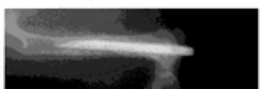

B
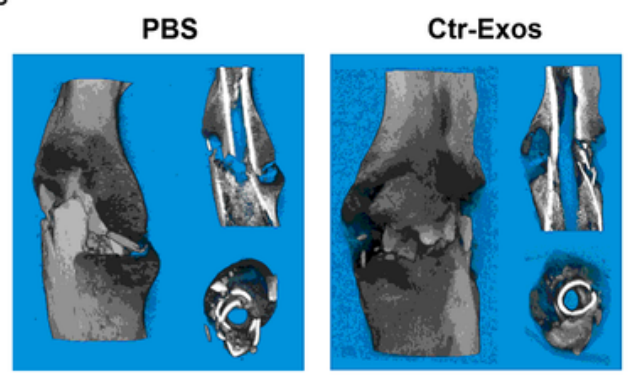

E

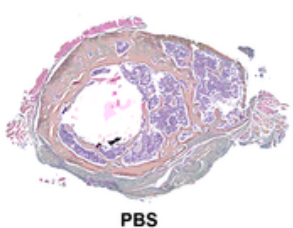

G
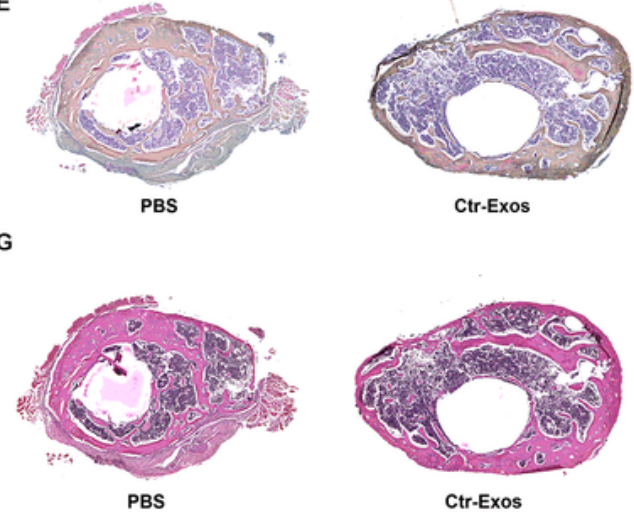

PBS

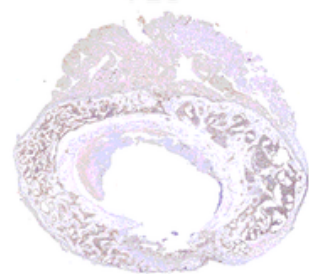

PBS

J
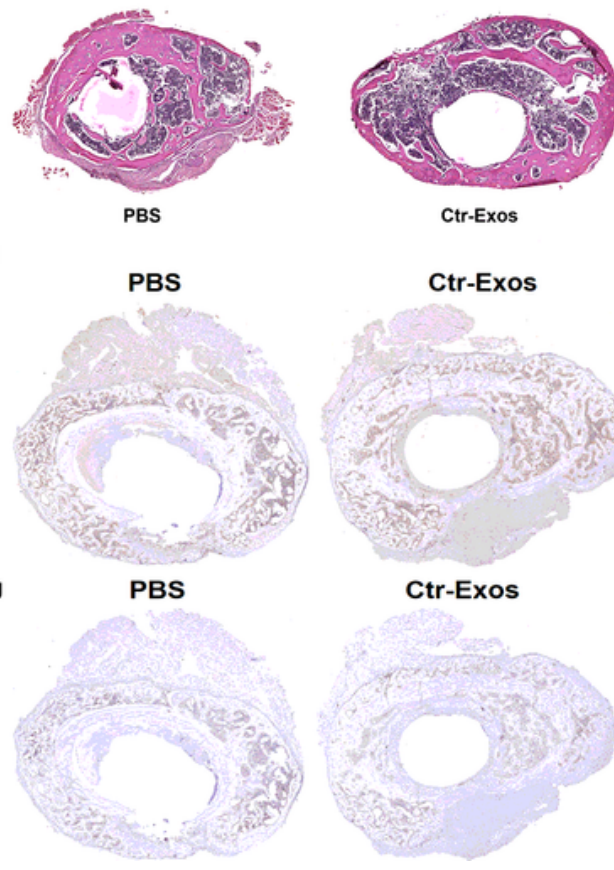

Ctr-Exos

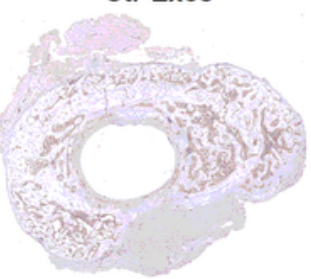

Ctr-Exos

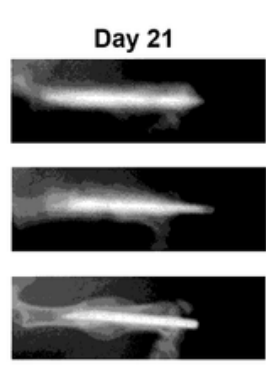

TBI-Exos

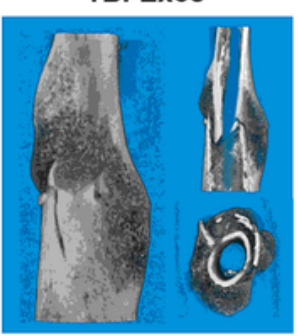

D
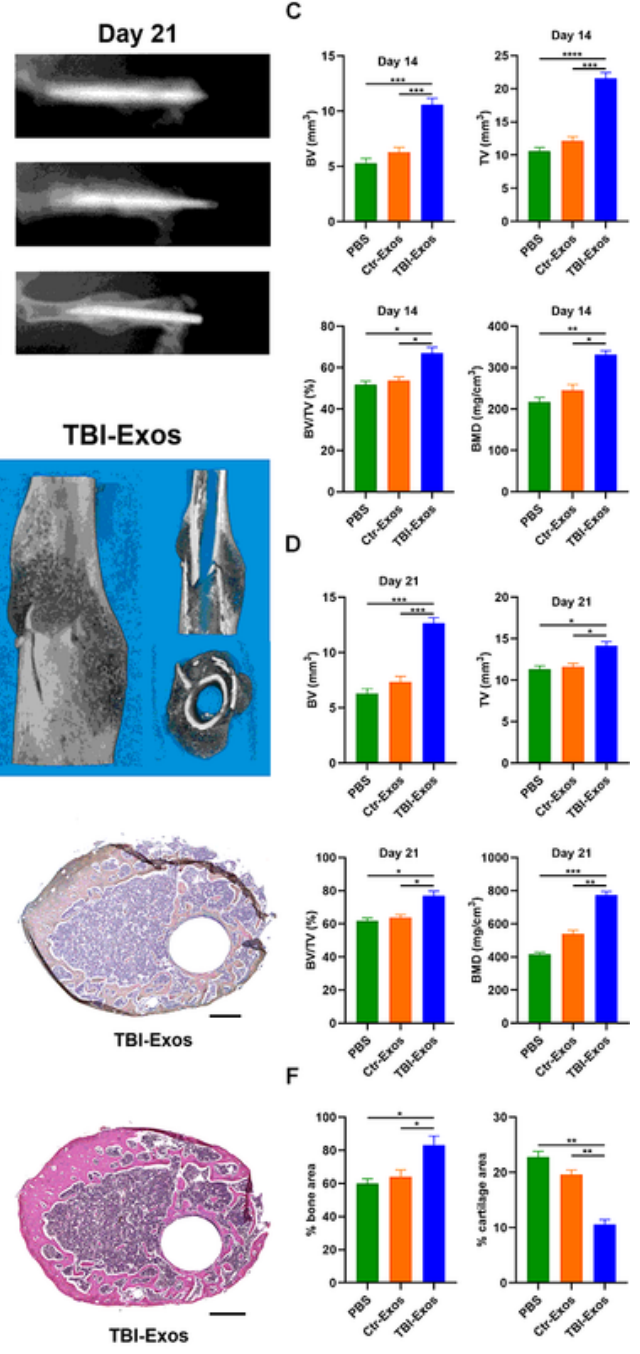

TBI-Exos

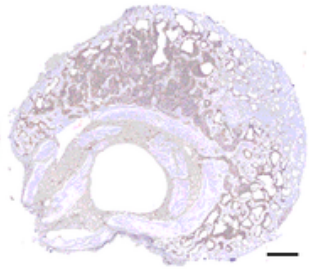

TBI-Exos

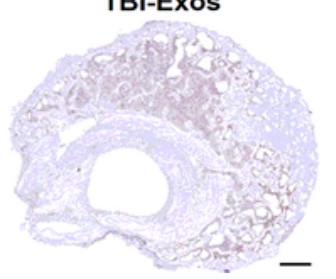

$\mathrm{H}$

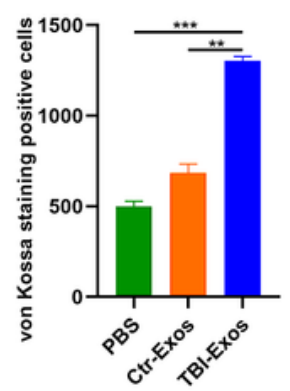

Figure 2 
TBI-Exos accelerates fracture healing in vivo. A-B. The healing processes in mice model with different treatments were assessed by X-rays (A) and micro-CT (B). C-D. The CT Analyser was used to measure the $B V, T V, B V / T V$ and BMD results of mice on day 14 and day 21 after fracture, respectively. E-H. The ABHEOG and Von Kossa staining results of mice on day 21 after fracture, scale bar: $200 \mu \mathrm{m}$. I-J, CD31 and Ki67 immunohistochemistry of the callus in different groups, scale bar: $500 \mu \mathrm{m}$.. Data are mean \pm SD of triplicate experiments. ${ }^{*} p<0.05,{ }^{*} \mathrm{p}<0.01,{ }^{* *} \mathrm{p}<0.001$.

Figure 3

A

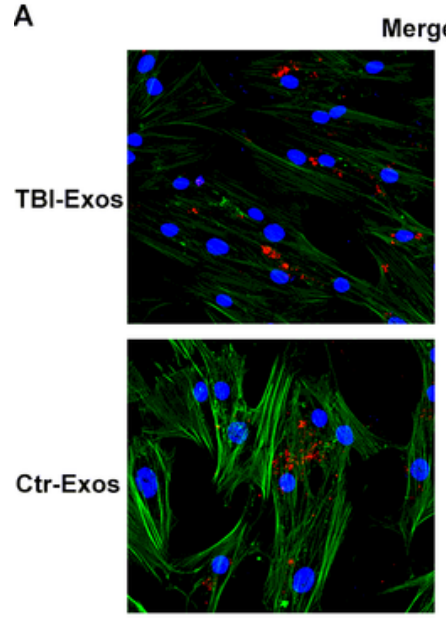

B

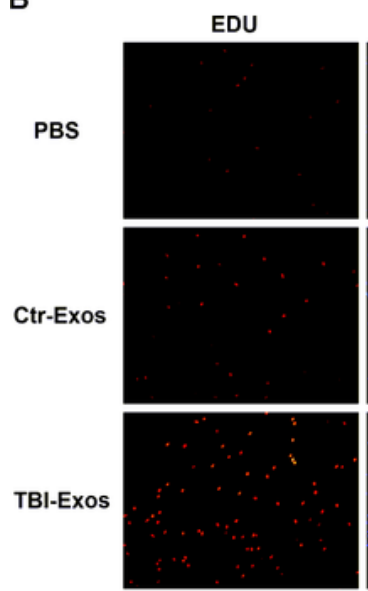

D

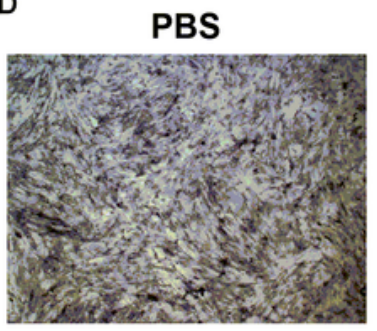

F

PBS

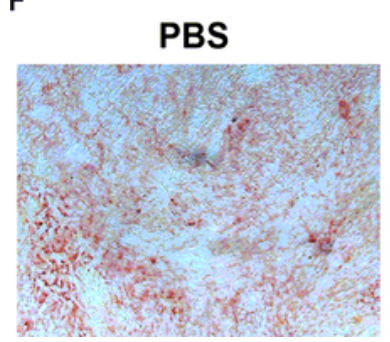

Ctr-Exos
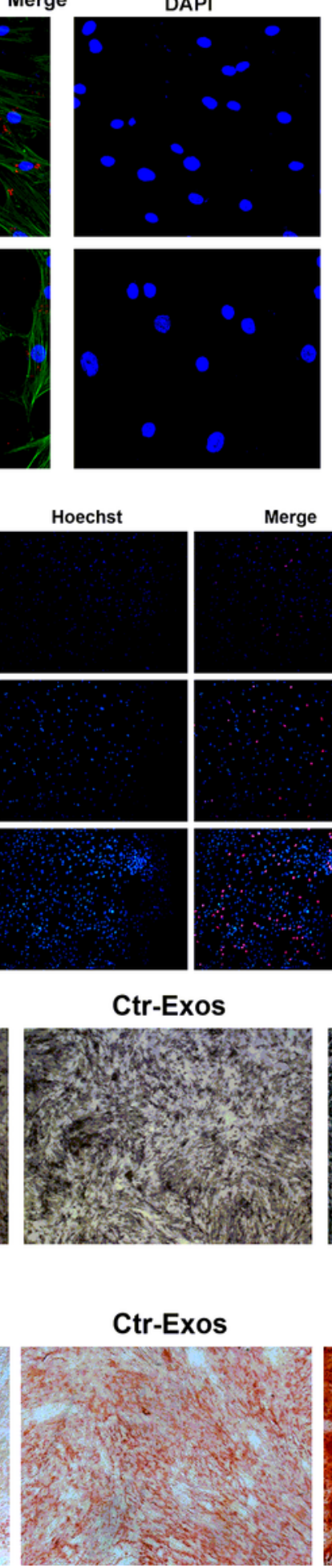

Ctr-Exos
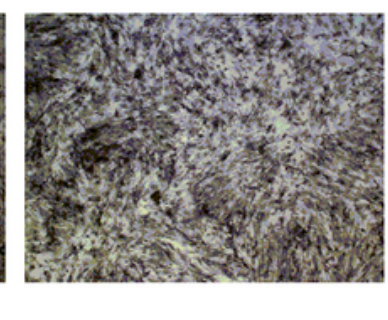

Figure 3
F-actin
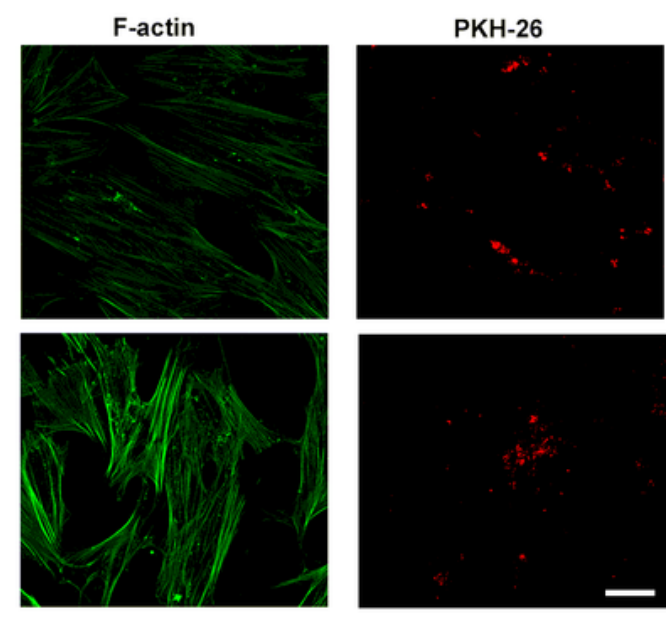

C

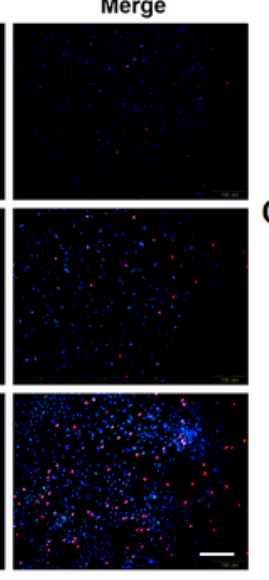

TBI-Exos

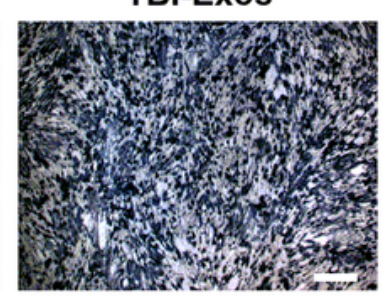

G

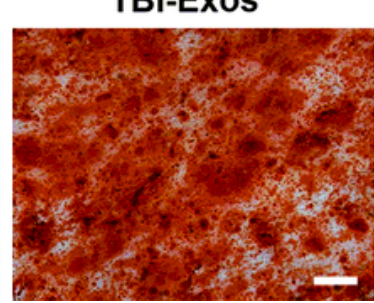

E
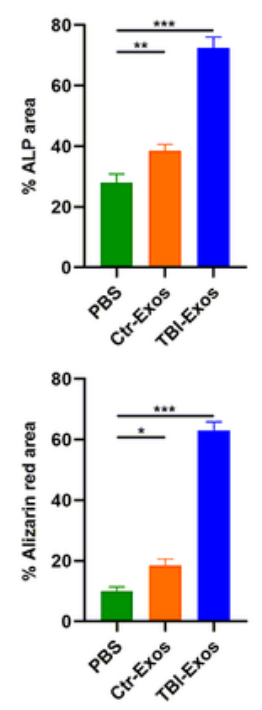
TBI-Exos promotes hMSCs proliferation and osteogenic differentiation. A. The uptake assay of Ctr-Exos and TBI-Exos in hMSCs. Scale bar: $50 \mu \mathrm{m}$. B. The Edu assay results of hMSCs with different treatments. Scale bar: $50 \mu \mathrm{m}$. C. The level of osteogenic-related proteins were measured by western blotting. D-E. The ALP staining result of hMSCs in the different groups. (F-G) The Alizarin Red staining result of hMSCs in the different groups. Data are mean $\pm S D$ of triplicate experiments. ${ }^{*} p<0.05,{ }^{* *} p<0.01,{ }^{* * *} p<0.001$.

A

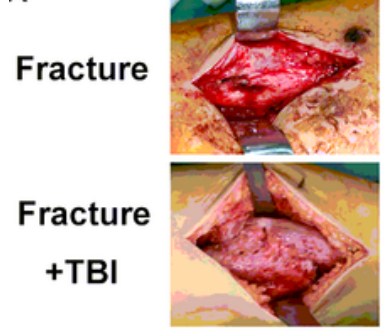

E

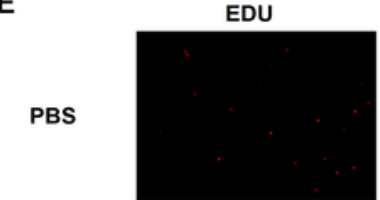

TBI-Exos
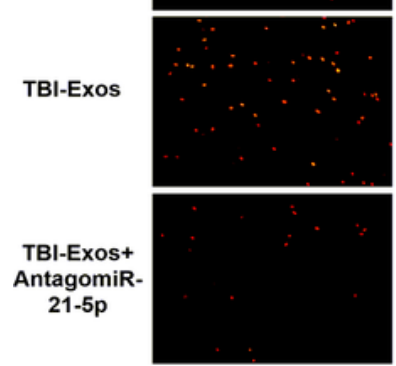

G

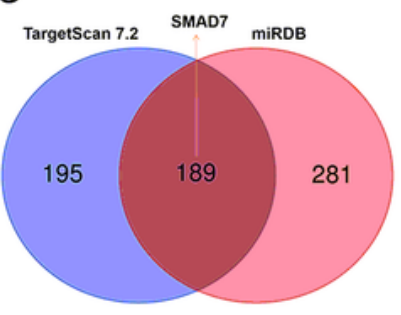

$\mathrm{K}$
B

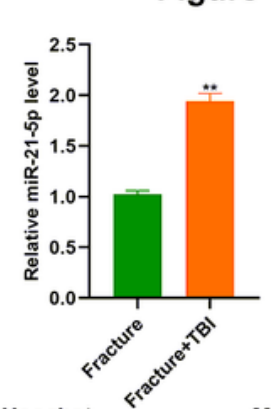

C

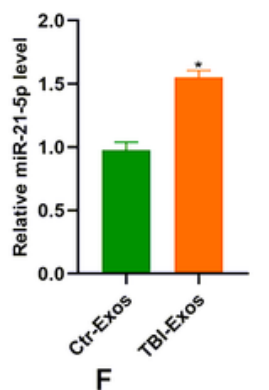

D

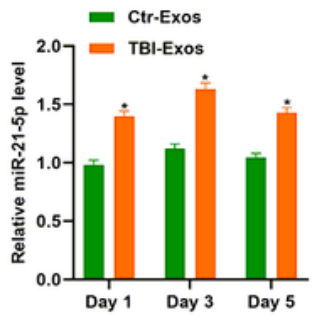

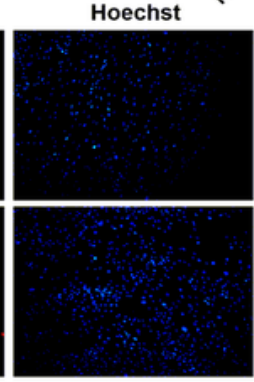

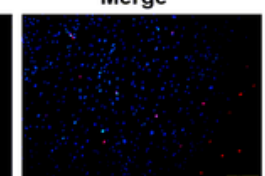

Collagen I
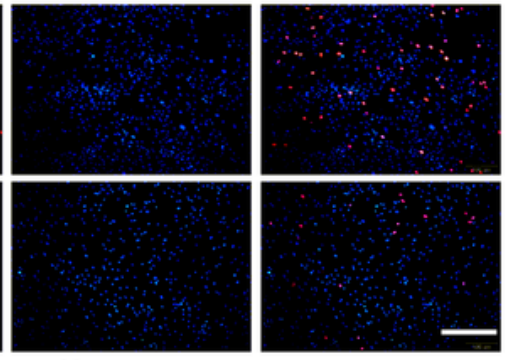

H

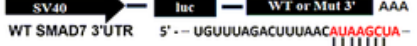
$\begin{array}{cc}\text { WT SMAD7 3'UTR } & 5^{\prime} \text { - - UGUUUAGACUUUAACAUAAGGCUA- } \\ \text { miR-21-5p } & 3^{\prime} \text { - - AGUUGUAGUCAGACUAUUCGAU }\end{array}$ MUT SMAD7 JUTR S'--UGUUUAGACUUUAACUAUUCGAA-
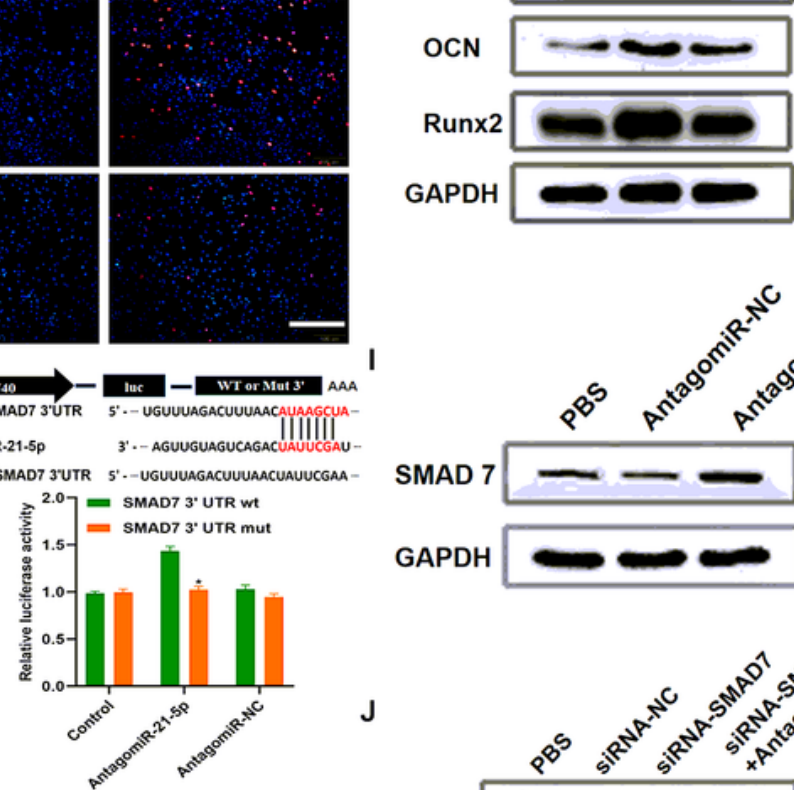

GAPDH
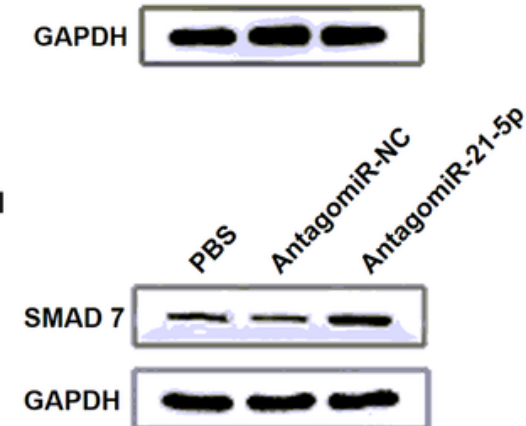

\section{J}
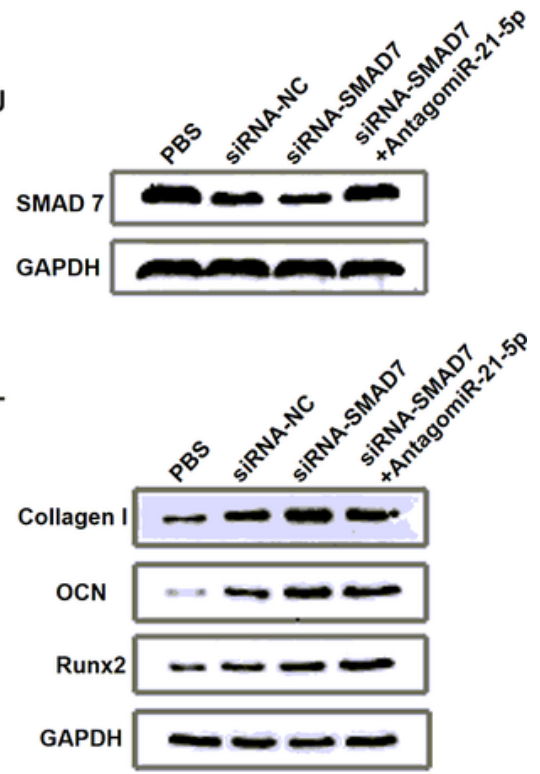

Figure 4 
The underlying mechanism of TBI in regulation of fracture healing. A. The calluses at the fracture site were more visible in fracture+TBI group when compared with those in the fracture group. B-C. The relative miR-21-5p level was measured both in serum and exos using qRT-PCR analysis. D. The miR-21-5p expression of hMSCs in different groups was measured by qRT-PCR analysis. E. EdU assay was performed to assess the cell proliferation in the different groups. $F$. The western blotting was used to detect the osteogenic-related proteins in the different groups. $G$, the potential targets of miR-21-5p were identified using the online predicting tools. I-J. SMAD7 level in the different groups was measured by western blotting analysis. K. The EdU assay was used to evaluated the hMSCs proliferation. L, the western blotting was used to measure the expression of osteogenic-related proteins. Data are mean $\pm S D$ of triplicate experiments. ${ }^{*} p<0.05,{ }^{* \star} p<0.01,{ }^{\star \star *} p<0.001$. 
A

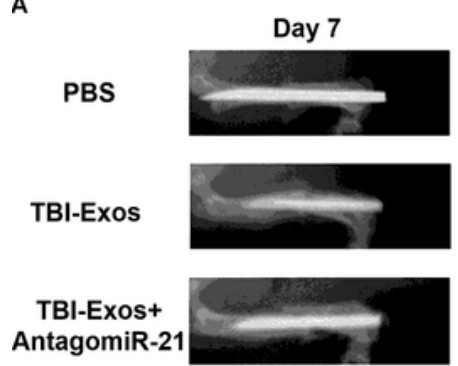

B

PBS

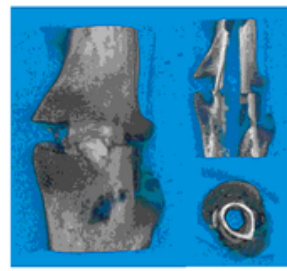

E

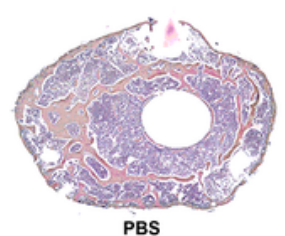

G

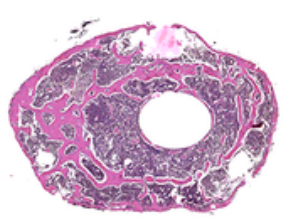

PBS

PBS

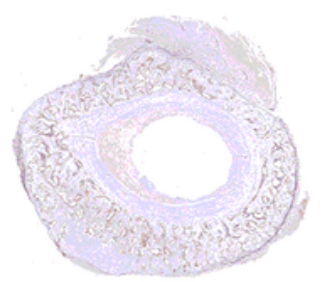

PBS
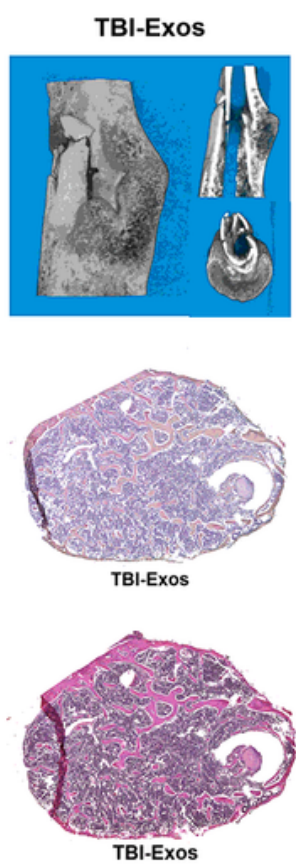

TBI-Exos

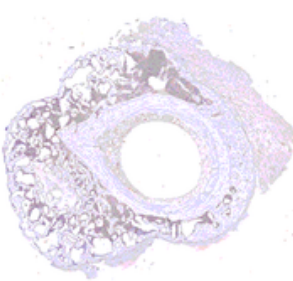

TBI-Exos

Fgure 5

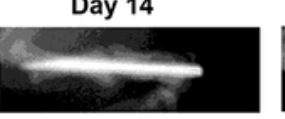

Day 21
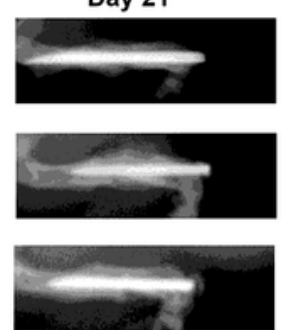

TBI-Exos+AntagomiR-21
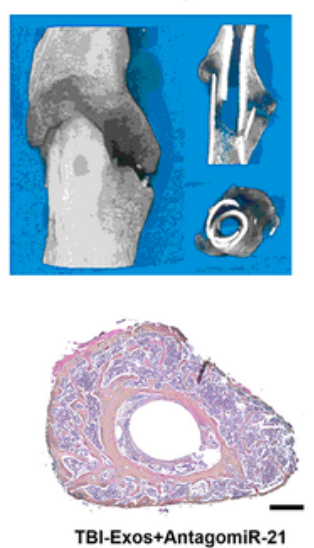

TBI-Exos+AntagomiR-21

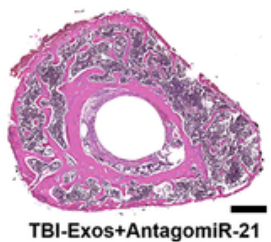

TBI-Exos+

AntagomiR-21-5p

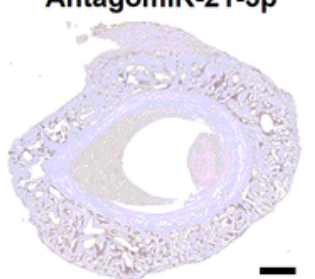

TBI-Exos+

AntagomiR-21-5p
C
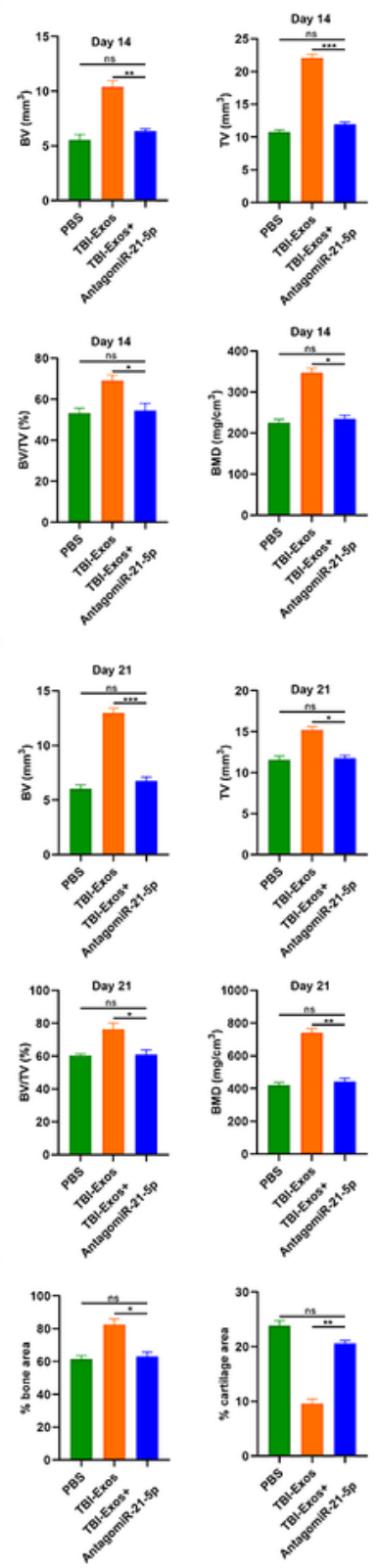

H

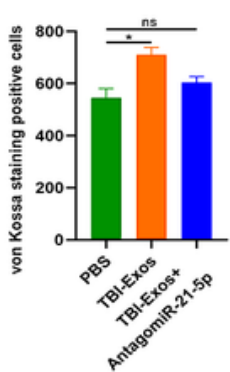

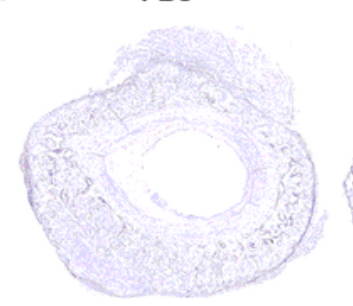
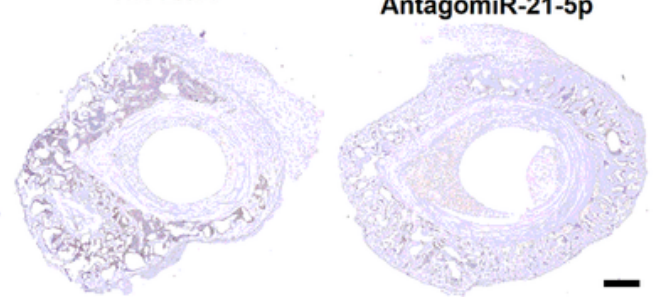

\section{Figure 5}

Exosomal miR-21-5p promotes fracture healing in vivo. A-B. The healing processes in mice model with different treatments were assessed by X-rays (A) and micro-CT (B). C-D. The CT Analyser was used to measure the BV, TV, BV/TV and BMD results of mice on day 14 and day 21 after fracture, respectively. E$\mathrm{H}$. The ABHEOG and Von Kossa staining results of mice on day 21 after fracture, scale bar: $200 \mu \mathrm{m}$. I-J, 
CD31 and Ki67 immunohistochemistry of the callus in different groups, scale bar: $500 \mu \mathrm{m}$.. Data are mean $\pm S D$ of triplicate experiments. ${ }^{*} p<0.05,{ }^{*} p<0.01,{ }^{* \star *} p<0.001$.

\section{Supplementary Files}

This is a list of supplementary files associated with this preprint. Click to download.

- Supplementaryinformation.docx 